13,03

\title{
Атомная и электронная структура реконструкций поверхности (111) в кристаллах ZnSe и CdSe
}

\author{
(C) В.Л. Бекенев, С.М. Зубкова \\ Институт проблем материаловедения им. И.Н. Францевича Национальной академии наук Украины, \\ Киев, Украина \\ E-mail: bekenev@materials.kiev.ua
}

(Поступила в Редакцию 20 апреля 2017 г.

В окончательной редакции 10 мая 2017 г.)

\begin{abstract}
Впервые проведены первопринципные расчеты атомной и электронной структуры четырех вариантов полярных поверхностей $\mathrm{ZnSe}$ и $\mathrm{CdSe}(111)-(2 \times 2)$, заканчивающихся катионом: идеальной, релаксированной, реконструированной и релаксированной после реконструкции. В приближении слоистой сверхрешетки поверхность моделировали пленкой толщиной 12 атомных слоев и вакуумным промежутком $\sim 16 \AA$. Для замыкания оборванных связей $\mathrm{Se}$ на противоположной стороне пленки добавлено четыре фиктивных атома водорода с зарядом 0.5 электрона каждый. Ab initio расчеты проводились с использованием программы QUANTUM ESPRESSO, основанной на теории функционала плотности. Показано, что релаксация приводит к расщеплению атомных слоев. Для четырех вариантов поверхности рассчитаны и проанализированы зонные структуры, а также полные и послойные плотности электронных состояний.
\end{abstract}

Все расчеты были выполнены на вычислительном кластере Института проблем материаловедения НАН Украины.

DOI: 10.21883/FTT.2018.01.45308.136

\section{1. Введение}

Полупроводники $\mathrm{A}^{2} \mathrm{~B}^{6}$ вызывают все больший интерес в связи с их широким применением в оптоэлектронных устройствах, в частности, для детектирования и стимуляции излучения в инфракрасной и видимой областях спектра [1-8].

$\mathrm{ZnSe}$ - широкозонный полупроводник с очень низким коэффициентом поглощения в интервале длин волн 0.63-18 $\mu \mathrm{m}$. Он является ключевым материалом для изготовления высокомощных лазеров, инфракрасных окон, линз и призм. Прогресс в конструировании светоизлучающих (лазерных) диодов на основе $\mathrm{ZnSe}$ для зеленоголубой области спектра [9-11] возродил интерес к фундаментальным свойствам этого материала. Успешное применение в фотонике подтолкнуло к масштабным экспериментальным и теоретическим исследованиям атомных, геометрических и электронных свойств его поверхностных слоев [12-20]. Благодаря $\mathrm{ZnSe}$ и родственным ему соединениям II-VI появились новые возможности для изготовления разбавленных магнитных полупроводников и интерфейсов ферромагнетик/соединение II-VI для создания устройств спинтроники.

В свою очередь, в последнее десятилетие также проводятся масштабные теоретические и экспериментальные исследования поверхностных структур в кристалле CdSe. Тонкие пленки CdSe применяются сегодня в качестве фотопроводников, солнечных элементов, тонкопленочных транзисторов, газовых сенсоров и т.д.

Работа [11] является одним из первых теоретических исследований по расчетам полной энергии релаксированной и реконструированной поверхности $\mathrm{ZnSe}(111)-(2 \times 2)$, оканчивающейся $\mathrm{Zn}$, с использо- ванием метода сильной связи. Было показано, что образование вакансии катиона является энергетически выгодным на поверхностях II-VI.

В [12] на основе теории функционала плотности в приближении локальной плотности (DFT-LDA) были выполнены расчеты $a b$ initio полной энергии (в зависимости от поверхностной стехиометрии и химического потенциала $\mathrm{Cd}$ ) поверхностей $\mathrm{CdSe}(001)$ и (111), оканчивающихся $\mathrm{Cd}$ и $\mathrm{Se}$, с возможными реконструкциями $(2 \times 1),(1 \times 2),(2 \times 2),(4 \times 2)$ и $(2 \times 4)$. Расчеты показали, что существуют Сd-димеры на поверхности (001) и Se-тетрамеры на поверхности (111). Было показано, что вакансия Сd в структуре $(111)(2 \times 2)$ энергетически выгодна в области высокого химического потенциала $\mu \mathrm{Cd}$, в то время как Se-тетрамер в структурах $(111)(2 \times 4)$ и $(2 \times 2)$ энергетически выгоден в области низкого $\mu \mathrm{Cd}$.

Расчеты атомной и электронной структур, в том числе зонной структуры и плотности электронных состояний в реконструированных поверхностях (111) ZnSe и $\mathrm{CdSe}$ нам неизвестны.

Отметим, что кристаллические поверхности (111) полярны, а потому плоскости (111) и ( $\overline{1} \overline{1} \overline{1})$ неэквивалентны (см. рис. 1).

Экспериментально и теоретически было показано, что реконструкции полярных поверхностей значительно различаются скоростью роста, качеством эпитаксиальных пленок, а также на атомном (структурном) уровне $[21,22]$.

\section{2. Метод расчета}

В настоящей работе, опираясь на результаты $[11,12]$, мы провели теоретическое исследование атомной и 
(111)A

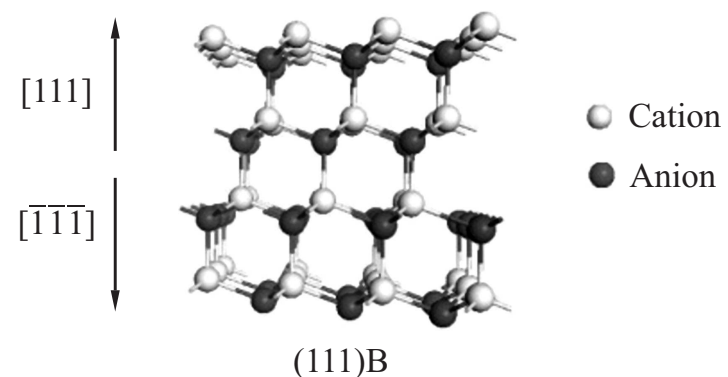

Рис. 1. Кристаллическая структура полярных поверхностей $\mathrm{A}^{2} \mathrm{~B}^{6}(111)$.

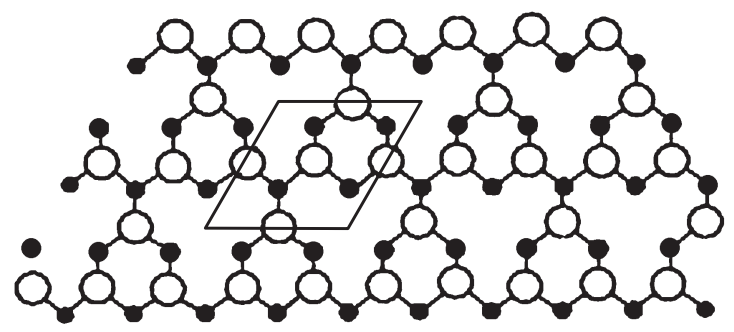

Рис. 2. Модель реконструкции $(2 \times 2)$ с вакансией цинка (кадмия). Белые кружки - $\mathrm{Zn}(\mathrm{Cd})$ в верхнем поверхностном слое, черные кружки - Se в подповерхностном слое.

электронной структуры четырех вариантов поверхностей $\mathrm{ZnSe}$ и $\mathrm{CdSe}(111) \mathrm{A}(2 \times 2)$, заканчивающихся цинком и кадмием: идеальной, релаксированной, реконструированной и реконструированной с последующей релаксацией. Такой подход позволил проследить влияние релаксации/реконструкции на атомную и электронную структуру в каждом конкретном случае. Расчеты проводились $a b$ initio методом псевдопотенциала с обменно-корреляционным функционалом в приближении локальной плотности (LDA) с использованием программного пакета QUANTUM ESPRESSO [23], основанного на теории функционала плотности (DFT). При этом использовалось приближение слоистой сверхрешетки. В этой модели поверхность представляется в виде системы тонких пленок (слэбов), периодически повторяющихся в направлении, перпендикулярном поверхности, и разделенных вакуумными промежутками.

В расчете использовалась также модель слэба, предложенная K. Shiraishi [24] специально для полярных полупроводниковых поверхностей. ${ }^{1}$ В таком слэбе заряд не интересующей нас поверхности (111)В, оканчивающейся Se, нейтрализуется путем насыщения ее оборванных связей атомами водорода с дробным зарядом. Это позволяет устранить ее влияние на противоположную,

\footnotetext{
${ }^{1}$ Атом селена имеет 6 валентных электронов, атом цинка (кадмия) - 2 валентных электрона. 8 электронов образуют 4 валентных связи, в каждую из которых селен отдает 1.5 электрона, а цинк (кадмий $)-0.5$ электрона. Чтобы замкнуть оборванные связи четырех ионов селена на поверхностях $\mathrm{ZnSe}(\mathrm{CdSe})(111) \mathrm{B}$, надо присоединить к ней четыре фиктивных атома водорода с зарядом 0.5 электрона.
}

исследуемую поверхность слэба, оканчивающуюся атомами цинка или кадмия.

Известно, что при реконструкции $(2 \times 2)$ происходит удвоение периода поверхностной элементарной ячейки по двум направлениям и замена одного из четырех атомов цинка (кадмия) вакансией (см. рис. 2).

В нашем случае слэб, моделирующий поверхность, состоял из двенадцати заполненных слоев и вакуумного промежутка, равного $\sim 16 \AA$. Большая удлиненная элементарная ячейка, которая в случае реконструкции $\mathrm{Zn}(\mathrm{Cd}) \mathrm{Se}(111) \mathrm{A}(2 \times 2)$ учетверяется, выбирается таким образом, что в двух измерениях она определяется самыми короткими векторами прямой решетки, т.е. векторами $(a \sqrt{2} / 2) \cdot 2$, где $a-$ постоянная решетки кристалла со структурой сфалерита. В третьем измерении выбирается длинная ось $c$, которая проходит в направлении [111] вдоль большой диагонали куба (таким способом кубическая структура сфалерита рассматривается в гексагональной установке) и охватывает двенадцать заполненных слоев и вакуумный промежуток. Чередующиеся атомные слои цинка (кадмия) и селена, перпендикулярные этой оси, имеют плоскую гексагональную решетку с параметром $a \sqrt{2}$. Выбор двенадцати атомных слоев гарантирует, что пленка содержит целое число неприводимых кристаллических слоев (в данном случае два). Нереконструированная суперячейка $(2 \times 2)$ из двенадцати слоев содержит 48 атомов (по четыре атома в каждом слое). С учетом $d$-электронов $\mathrm{Zn}(\mathrm{Cd})$ число валентных электронов равно 432. В случае реконструкции в верхнем двенадцатом слое вместо одного атома цинка (кадмия) появляется вакансия. Такая элементарная ячейка содержит 47 атомов. В этом случае число валентных электронов в реконструированных ячейках $\mathrm{ZnSe}$ и $\mathrm{CdSe}$ равно 420. Для замыкания оборванных связей $\mathrm{Se}$ добавляются четыре фиктивных атома водорода с зарядом 0.5 электрона каждый. Таким образом, получаем 434 валентных электрона в случае отсутствия реконструкции и 422 валентных электрона в случае реконструкции.

Самосогласованные расчеты проводились с использованием сетки $\mathbf{k}$-точек $6 \times 6 \times 1$. После ряда тестовых расчетов энергия обрезания была взята равной $480 \mathrm{eV}$, что соответствует разложению волновой функции по $\sim 60000$ плоских волн. Оптимизация атомной структуры проводилась только для четырех верхних слоев (как в случае нереконструированной, так и реконструированной поверхностей). Положения атомов в этих слоях считались полностью релаксированными, когда силы, действующие на атомы, становились меньше $0.02 \mathrm{eV} / \AA$. Атомы в остальных слоях занимали те же позиции, что и в объеме образца.

\section{3. Атомная структура поверхности. Релаксация. Реконструкция}

Равновесные параметры решетки для $\mathrm{ZnSe}$ и $\mathrm{CdSe}$ со структурой сфалерита получены авторами с помощью расчета зависимости полной энергии от параметра решетки. Для $\mathrm{ZnSe} a=5.5763 \AA$, для $\mathrm{CdSe}$ 
$a$
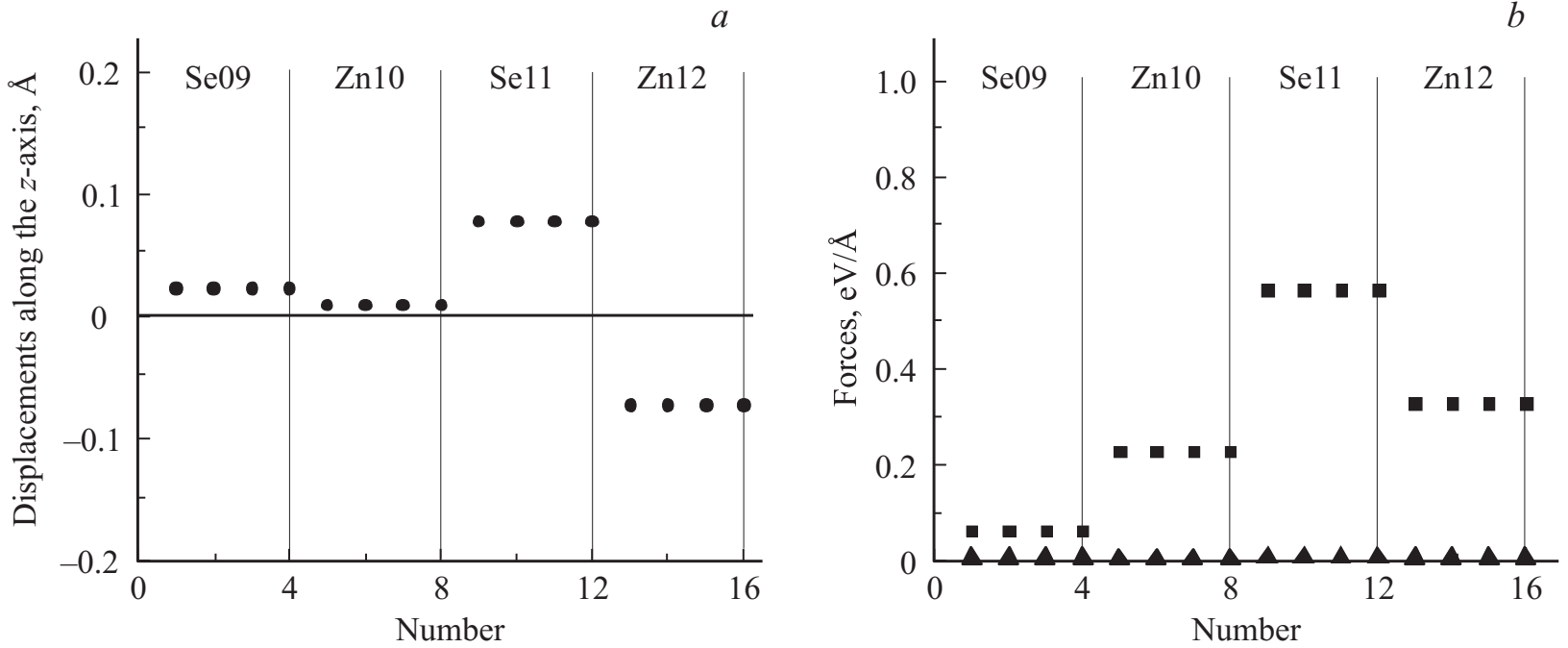

Рис. 3. Смещения атомов в верхних четырех слоях идеального слэба $\mathrm{ZnSe}$ вдоль координатной оси $z(a)$ и силы, действующие на атомы $(b)$ : квадраты - до релаксации, треугольники - после релаксации.
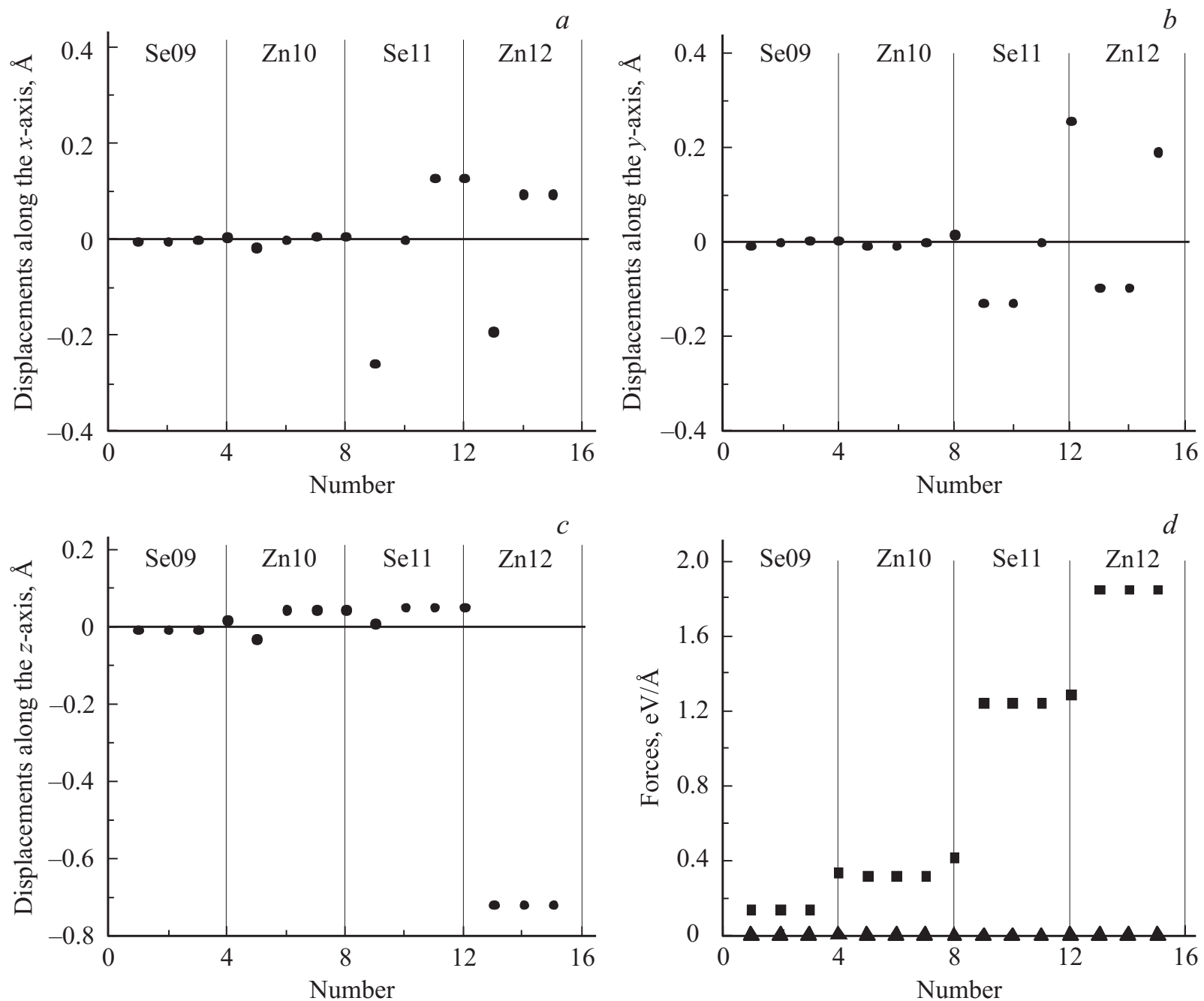

Рис. 4. Смещения атомов в верхних четырех слоях реконструированного слэба ZnSe вдоль координатных осей ( $a-c)$ и силы, действующие на атомы $(d)$ : квадраты - до релаксации, треугольники - после релаксации. 


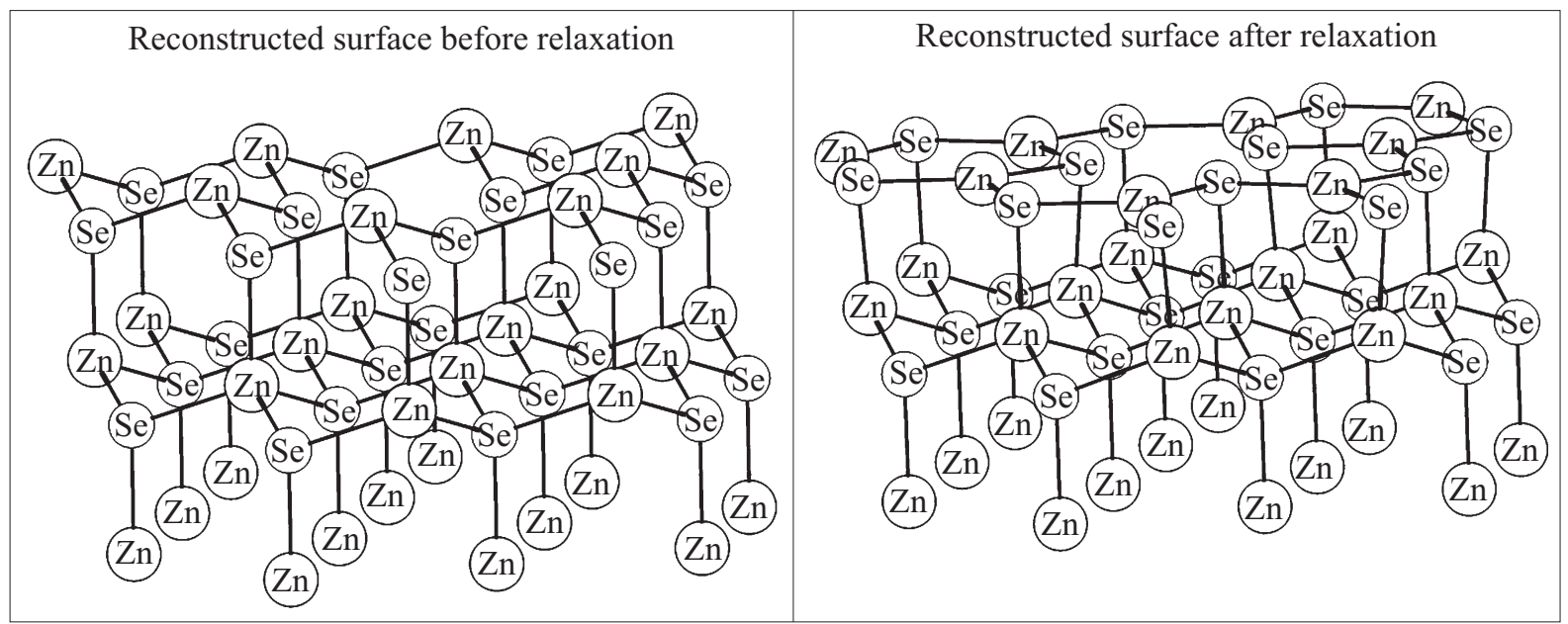

Рис. 5. Вид сбоку на пять верхних слоев слэба ZnSe в исходной реконструированной структуре и в реконструированной структуре после релаксации.
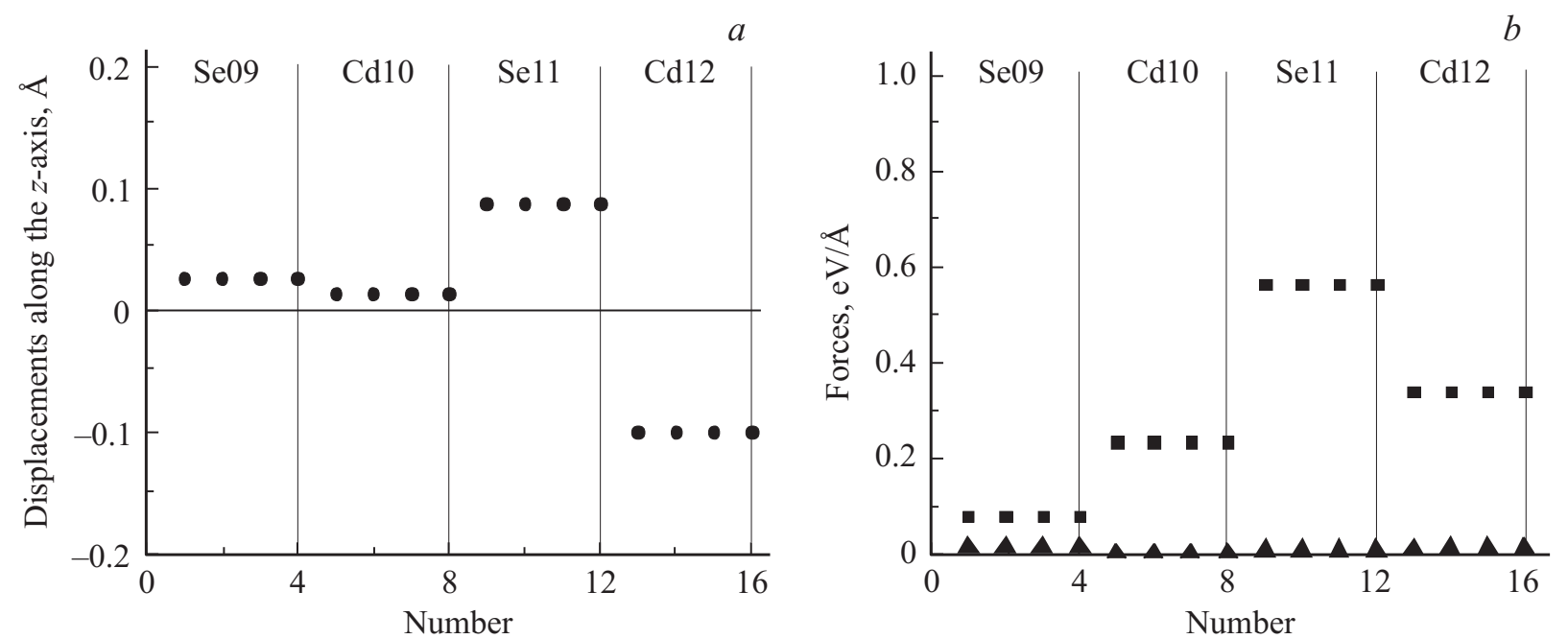

Рис. 6. Смещения атомов в верхних четырех слоях идеального слэба CdSe вдоль координатной оси $z(a)$ и силы, действующие на атомы $(b)$ : квадраты - до релаксации, треугольники - после релаксации.

$a=6.0053 \AA$ А. Рассчитанные параметры решетки хорошо согласуются с экспериментальными данными: так для $\mathrm{ZnSe} a=5.668 \AA$, для CdSe $a=6.052 \AA[25]$.

3.1. ZnSe. Для нереконструированной поверхности исследуемого кристалла релаксация приводит только к изменению расстояний между атомными слоями. Внутри же слоя атомы практически не смещаются.

На рис. 3, a приведен график величин смещений атомов вдоль оси $z$ после релаксации. График состоит из четырех частей: 1) слой $\mathrm{Se} 9$, номера атомов 1-4, 2) слой Zn10, атомы 5-8, 3) слой Se11, атомы 9-12, 4) слой $Z n 12$, атомы 13-16. Вдоль оси $z$ смещения в пределах каждого слоя практически одинаковы для всех атомов, при этом расстояние между подповерхностным слоем Se11 и поверхностным слоем Zn12 уменьшается с $0.805 \AA$ в идеальном слэбе до $0.657 \AA$. На рис. $3, b$ показаны силы, действующие на атомы до и после релаксации. Видно, что после релаксации силы во всех четырех слоях резко уменьшаются до тысячных долей $\mathrm{eV} / \AA \AA$. Отметим, что в идеальной суперячейке $\mathrm{ZnSe}$ максимальная сила действует на атомы селена в подповерхностном слое и равна $0.566 \mathrm{eV} / \AA$, а после релаксации она составляет $0.005 \mathrm{eV} / \AA$. Таким образом, релаксация идеального слэба более чем в 105 раз уменьшила максимальную силу. В случае просто реконструкции максимальная сила величиной $1.85 \mathrm{eV} / \AA$ действует на атомы цинка в поверхностном слое, а после релаксации она уменьшается до $0.005 \mathrm{eV} / \AA$.

На рис. 4, $a-d$ приведены графики величин смещений атомов в реконструированной пленке до и после релаксации в каждом из атомов верхних четырех слоев. Графики построены по тому же принципу, что и на рис. 3. В плоскости $x y$ атомы в слоях $\mathrm{Se} 9$ и $\mathrm{Zn} 10$ смещены незначительно, в слоях Se11 и Zn12 смещения существенно больше. Вдоль оси $z$ слои 9, 

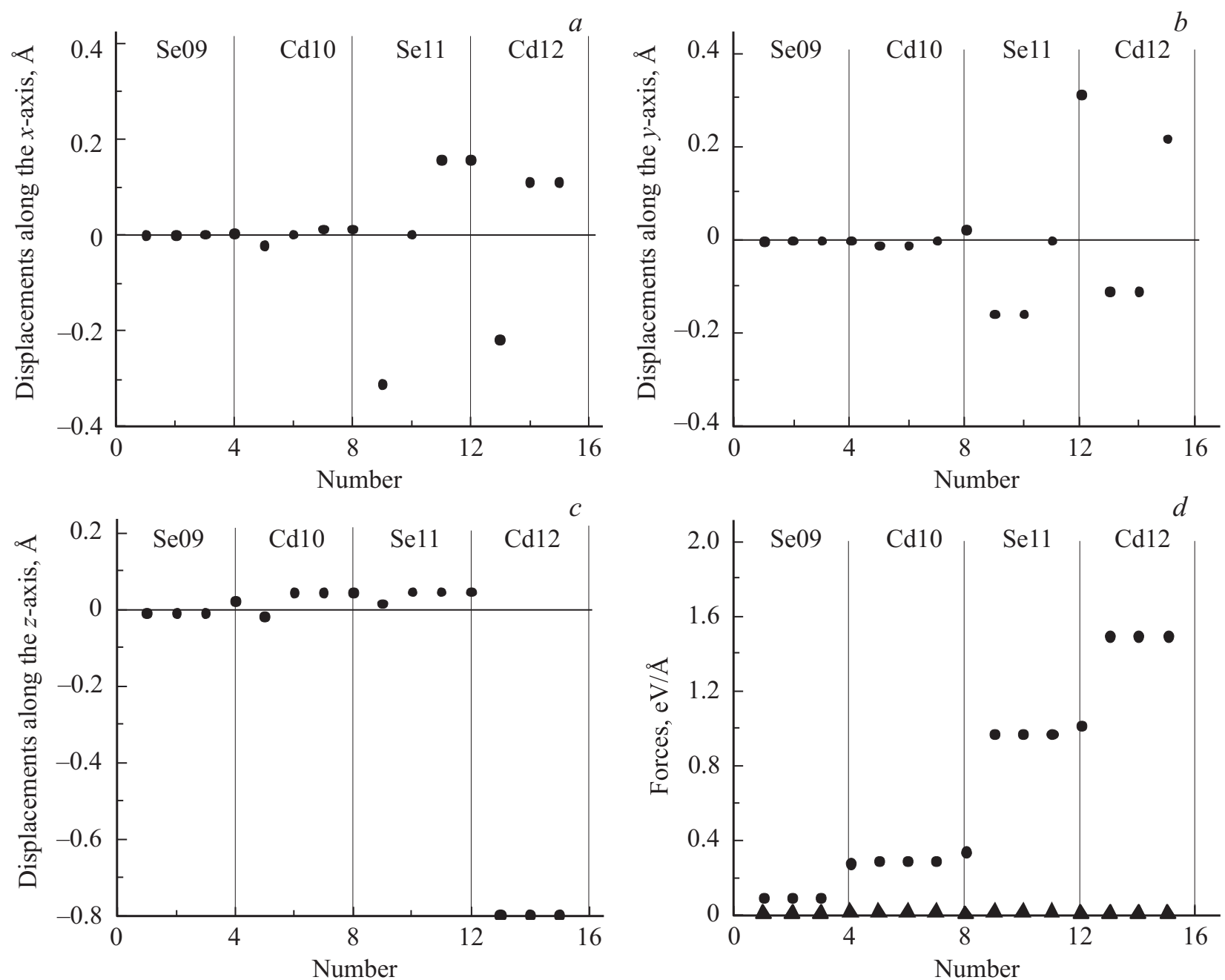

Рис. 7. Смещения атомов в верхних четырех слоях реконструированного слэба CdSe вдоль координатных осей $(a-c)$ и силы, действующие на атомы $(d)$ : квадраты - до релаксации, треугольники - после релаксации.

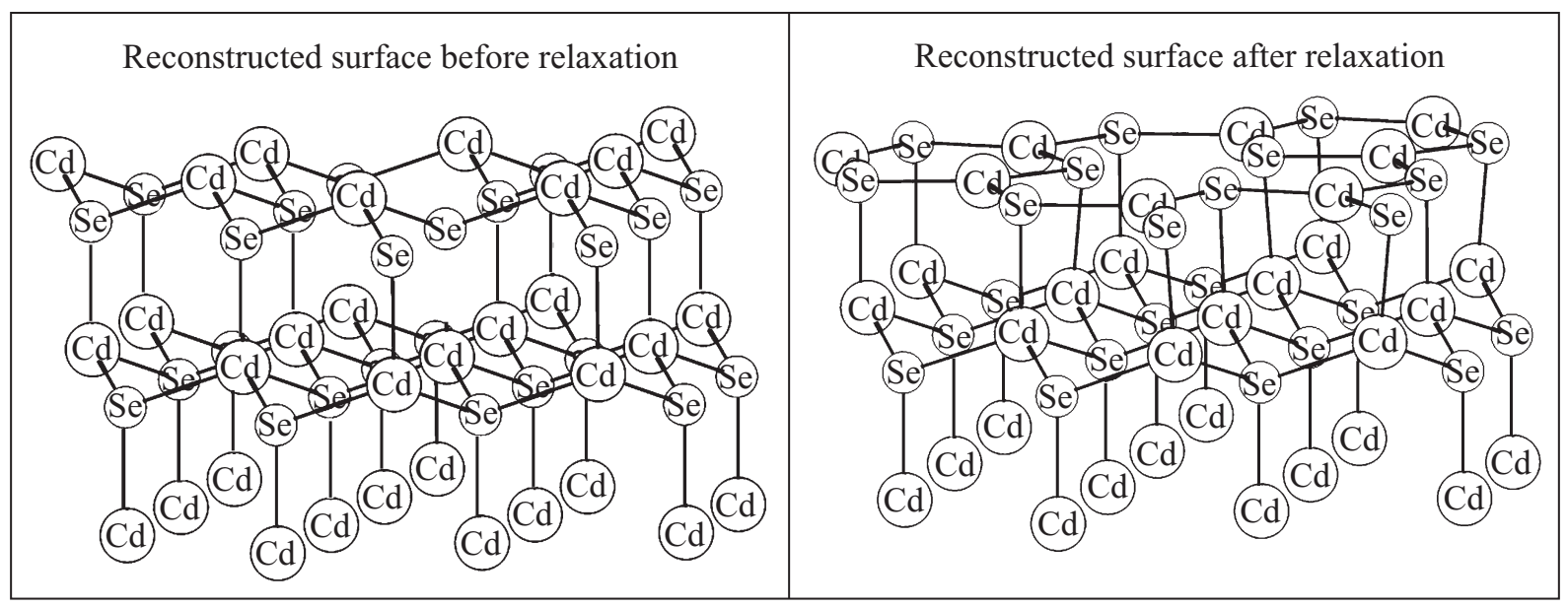

Рис. 8. Вид сбоку на пять верхних слоев слэба CdSe в реконструированной структуре до и после релаксации. 


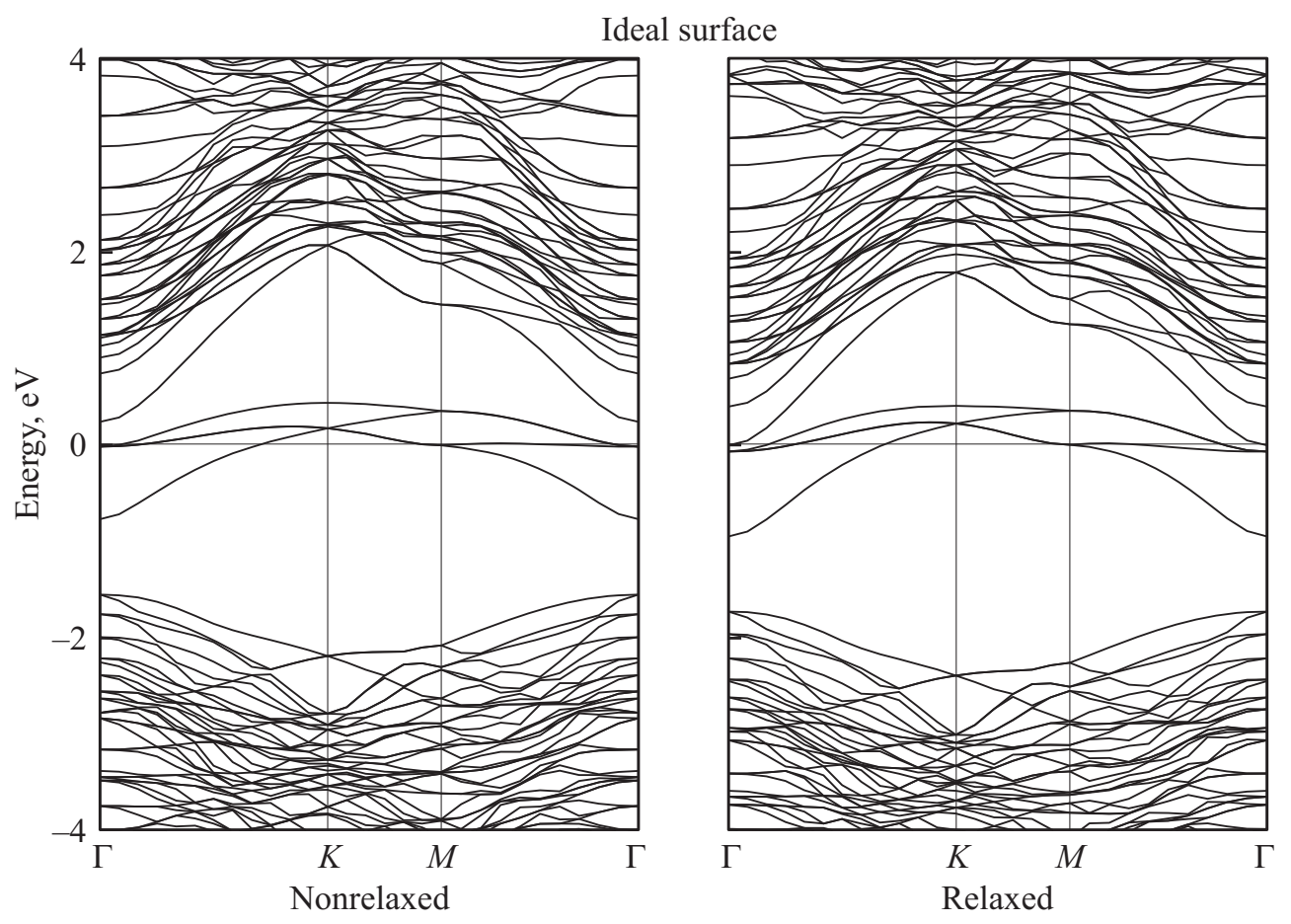

Рис. 9. Зонная структура идеальной поверхности (111) ZnSe без учета и с учетом релаксации.
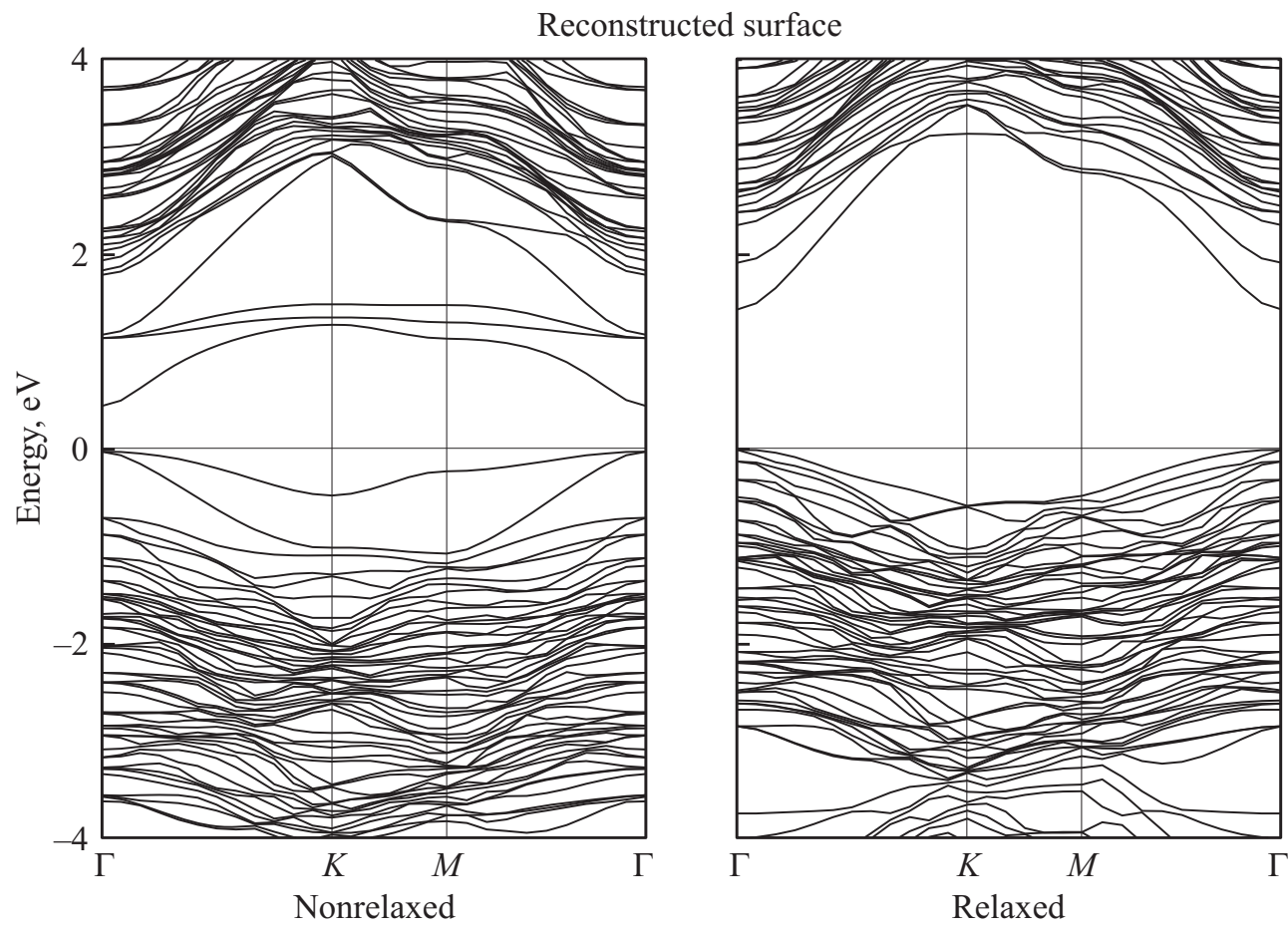

Рис. 10. Зонная структура реконструированной поверхности (111) ZnSe без учета и с учетом релаксации.

10 и 11 расщепились каждый на два слоя, один из которых содержит три атома, а другой - один атом. Величина расщепления находится в пределах $0.05 \AA$. Соответственно силы, действующие на атомы в слоях 9 , 10 и 11, тоже разделились на две группы в каждом слое. В то же время поверхностный слой Zn12 опустился на $0.72 \AA$ и оказался почти на том же уровне, что и подповерхностный слой $\mathrm{Se} 11$. Из рис. $4, d$ видно, что в поверхностном слое на все три атома действуют максимальные силы, равные $1.85 \mathrm{eV} / \AA$. После проведения релаксации реконструированного слэба силы резко уменьшились практически до нуля. 


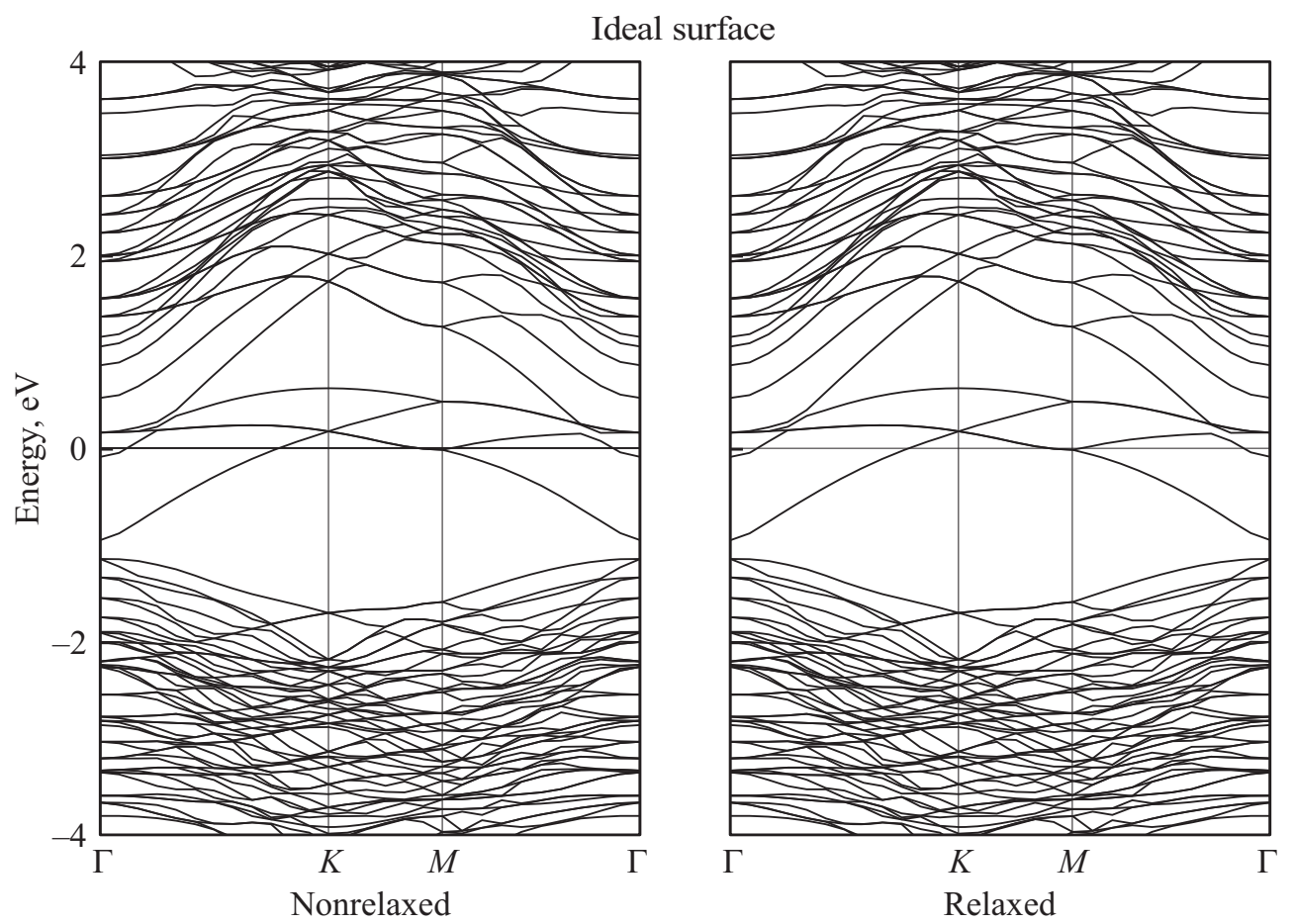

Рис. 11. Зонная структура идеальной поверхности (111) CdSe без учета и с учетом релаксации.

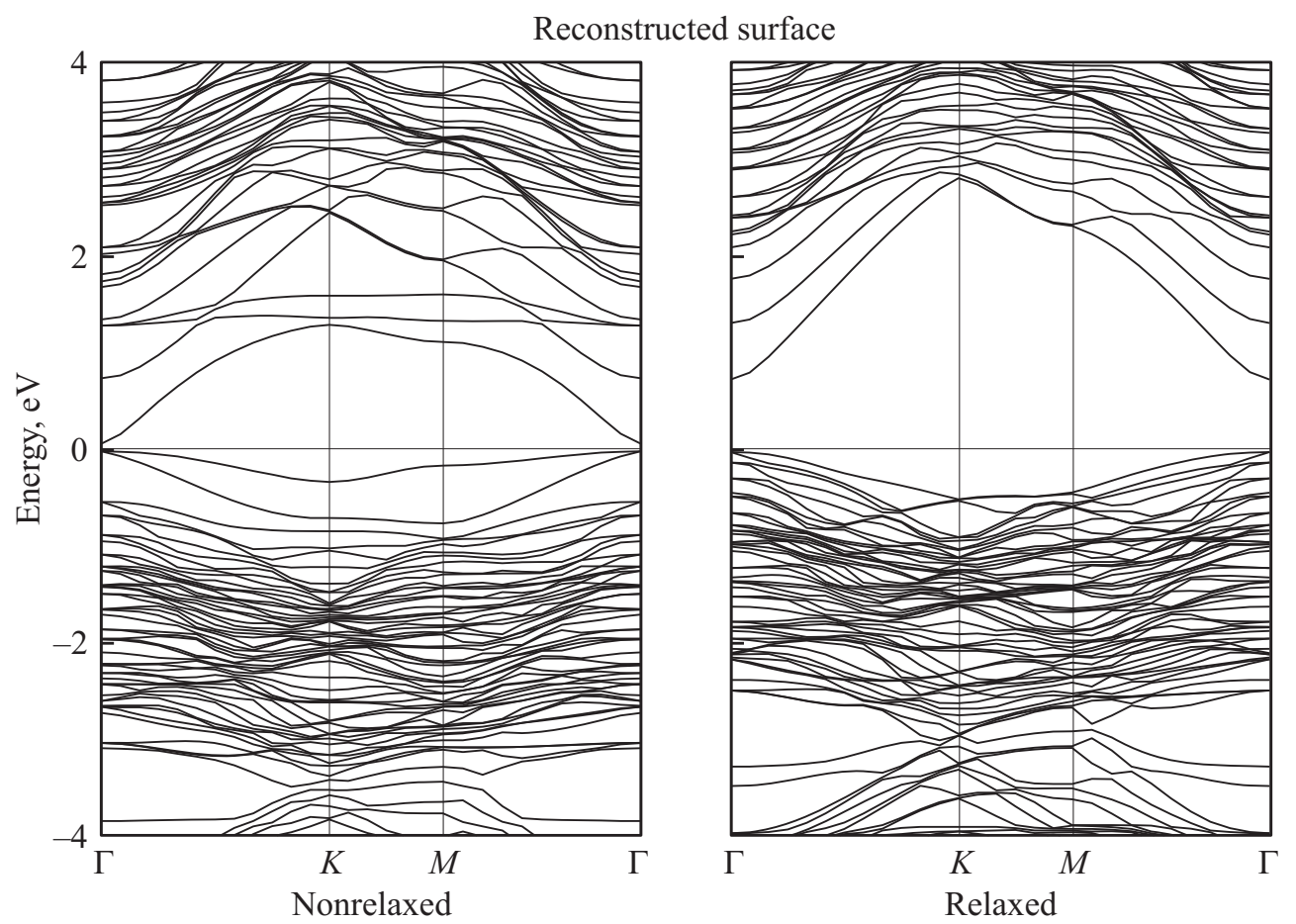

Рис. 12. Зонная структура реконструированной поверхности (111) CdSe без учета и с учетом релаксации.

Таким образом, в результате реконструкции/релаксации атомная структура пленки $\mathrm{ZnSe}$ существенно изменилась: три верхних атомных слоя $(9,10$ и 11) расщепились каждый на два слоя, а поверхностный слой опустился на уровень подповерхностного. Эти изменения хорошо видны на рис. 5, где представлен вид пяти верхних атомных слоев реконструированной и реконструированной/релаксированной поверхностей.

3.2. CdSe. Расчет смещений атомов в верхних четыpex слоях после релаксации вдоль осей $x, y$ и $z$ показал, 


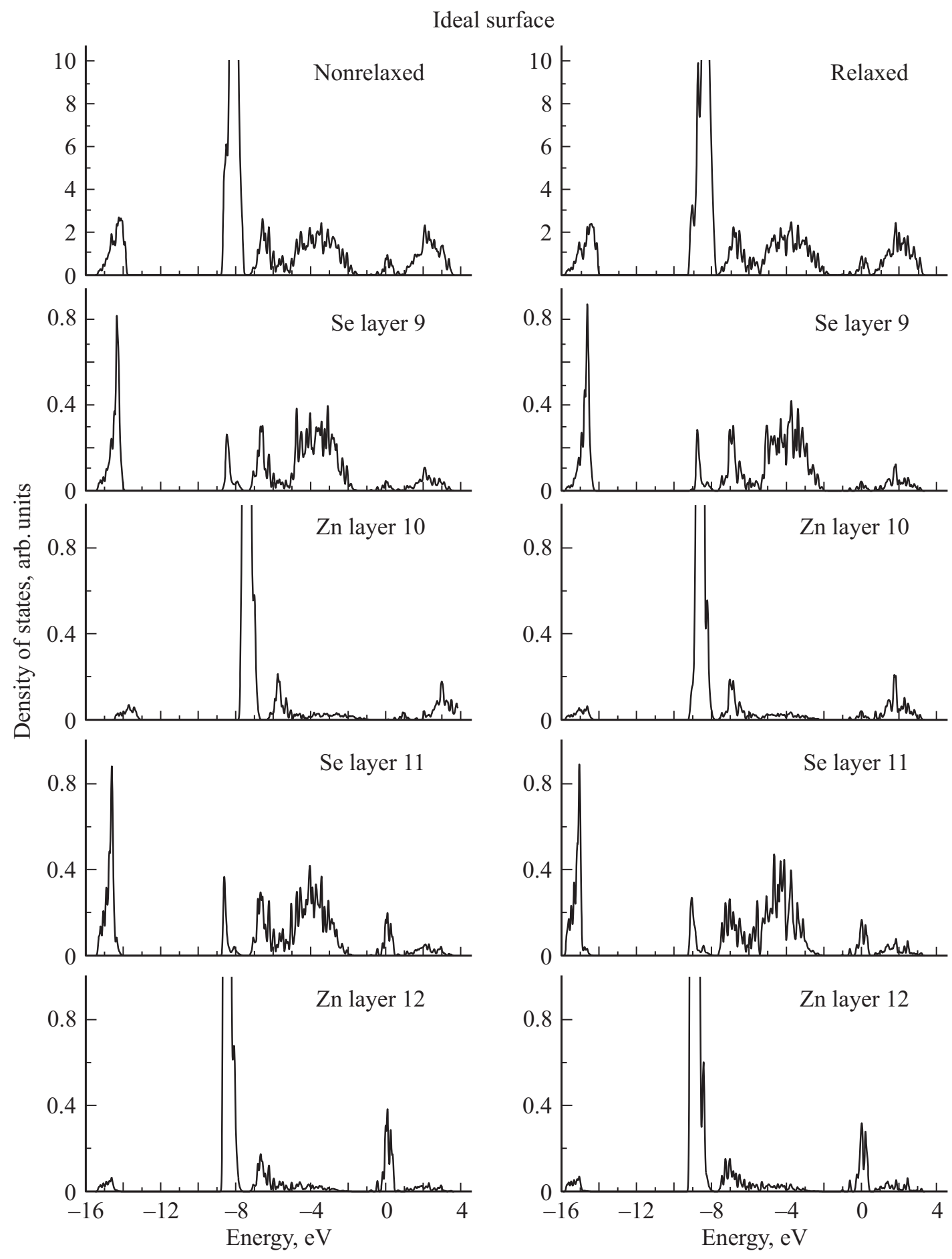

Рис. 13. Полная и послойная DOS идеальной поверхности (111) ZnSe без учета и с учетом релаксации.

что атомы вдоль осей $x, y$ практически не смещаются. На рис. 6, $a$ приведен график величин смещений атомов вдоль оси $z$ после релаксации. Описание структуры графика приведено в разделе 3.1.

Вдоль оси $z$ смещения в пределах каждого слоя практически одинаковы для всех атомов. Подповерхностный слой Se11 смещается вверх по оси $z$, а слой $\mathrm{Cd} 12$ - вниз. Расстояние между ними умень- шается почти на 20\%. На рис. 6, $b$ показаны силы, действующие на атомы до и после релаксации. Отметим, что в идеальной суперъячейке $\mathrm{CdSe}$ максимальная сила действует на атом в подповерхностном слое Se11 и равна $0.562 \mathrm{eV} / \AA \AA$, а после релаксации максимальная сила действует в слое $\mathrm{Cd} 10$ и составляет $0.0016 \mathrm{eV} / \AA ̊$. Таким образом, релаксация идеального слэба более чем в 350 раз уменьшила максимальную силу. Численный 
Reconstructed surface
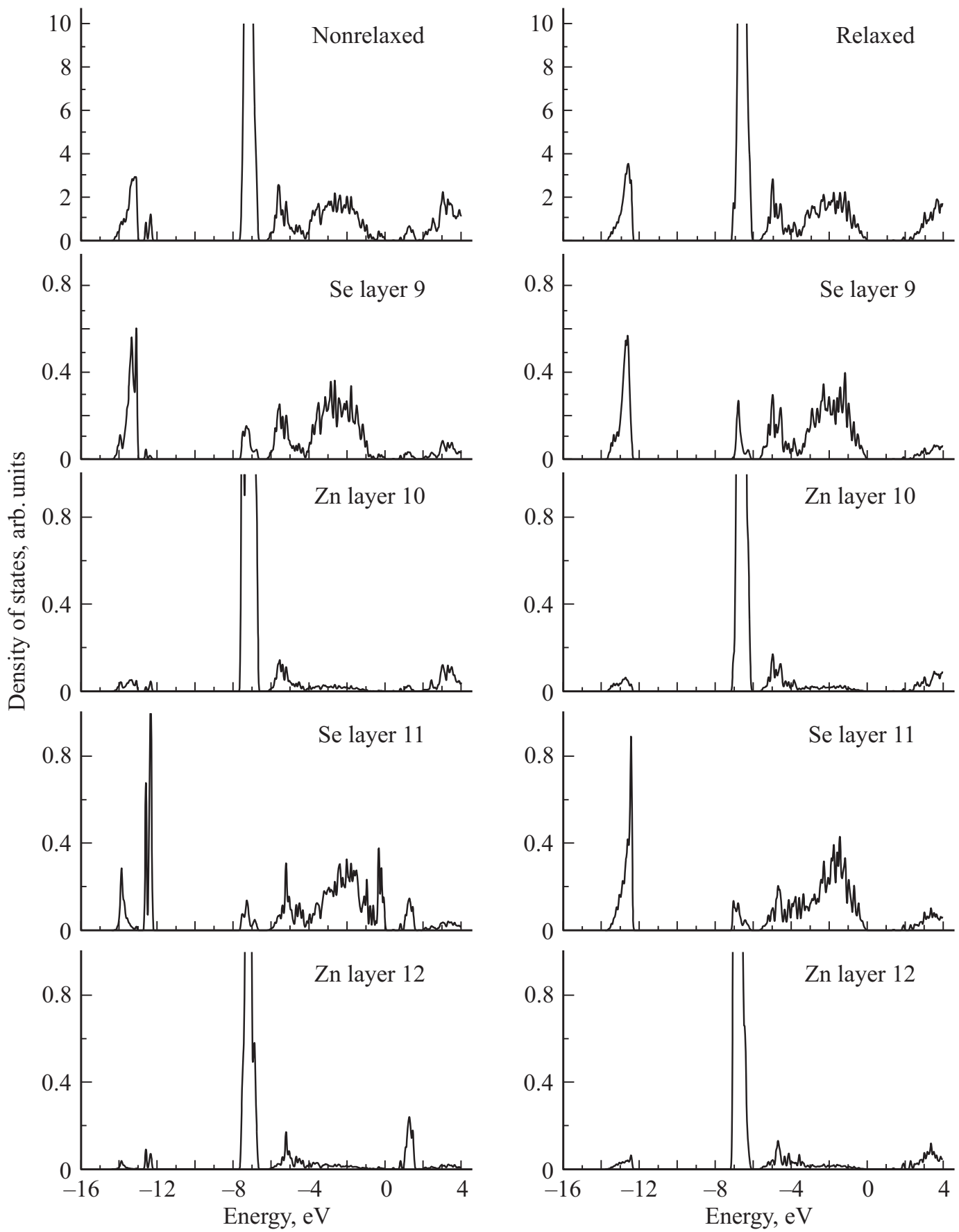

Рис. 14. Полная и послойная DOS реконструированной поверхности (111) ZnSe без учета и с учетом релаксации.

расчет показал, ${ }^{2}$ что после релаксации силы во всех четырех слоях резко уменьшаются до сотых-тысячных долей $\mathrm{eV} / \AA$.

На рис. 7, $a-d$ приведены графики величин смещений атомов в реальной поверхности (после реконструкции и релаксации) для каждого из атомов верхних четырех

\footnotetext{
${ }^{2}$ Авторы располагают численной информацией о координатах и величинах смещений атомов и о силах, действующих на атомы, в виде таблиц и готовы предоставить их по запросу читателя.
}

слоев. Графики построены по тому же принципу, что и на рис. 6. В плоскости $x y$ атомы в слоях $\mathrm{Se} 9$ и Cd10 смещены незначительно, в слоях Se11 и Cd12 смещения существенно больше. Вдоль оси $z$ слои 9 , 10 и 11 расщепились каждый на два слоя, один из которых содержит три атома, а другой - один атом. Величина расщепления находится в пределах $0.05 \AA$. Соответственно силы, действующие на атомы в слоях 9 , 10 и 11, тоже разделились на две группы в каждом слое. 


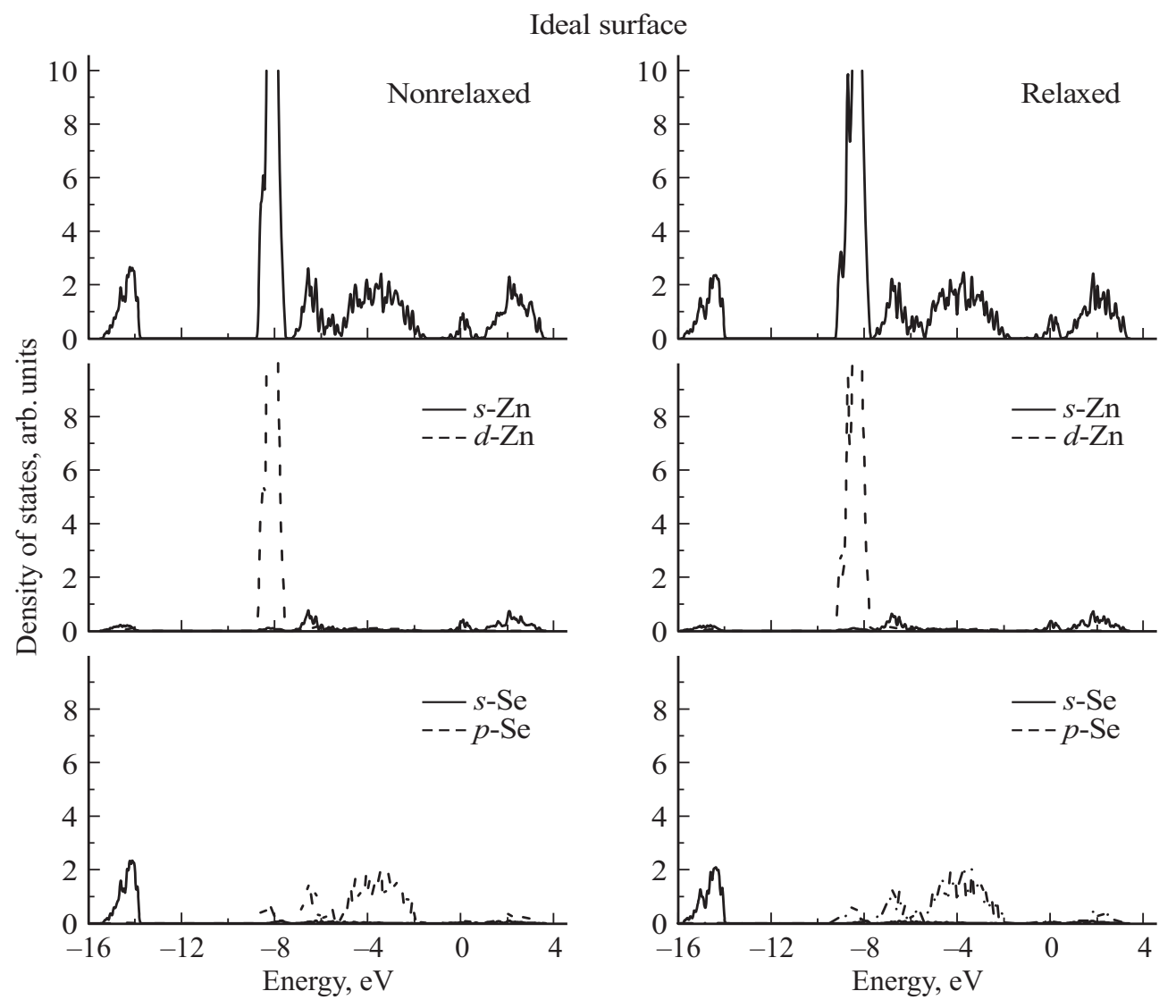

Рис. 15. Полная и $s-, p$-, $d$-DOS идеальной поверхности (111) ZnSe без учета и с учетом релаксации. Reconstructed surface
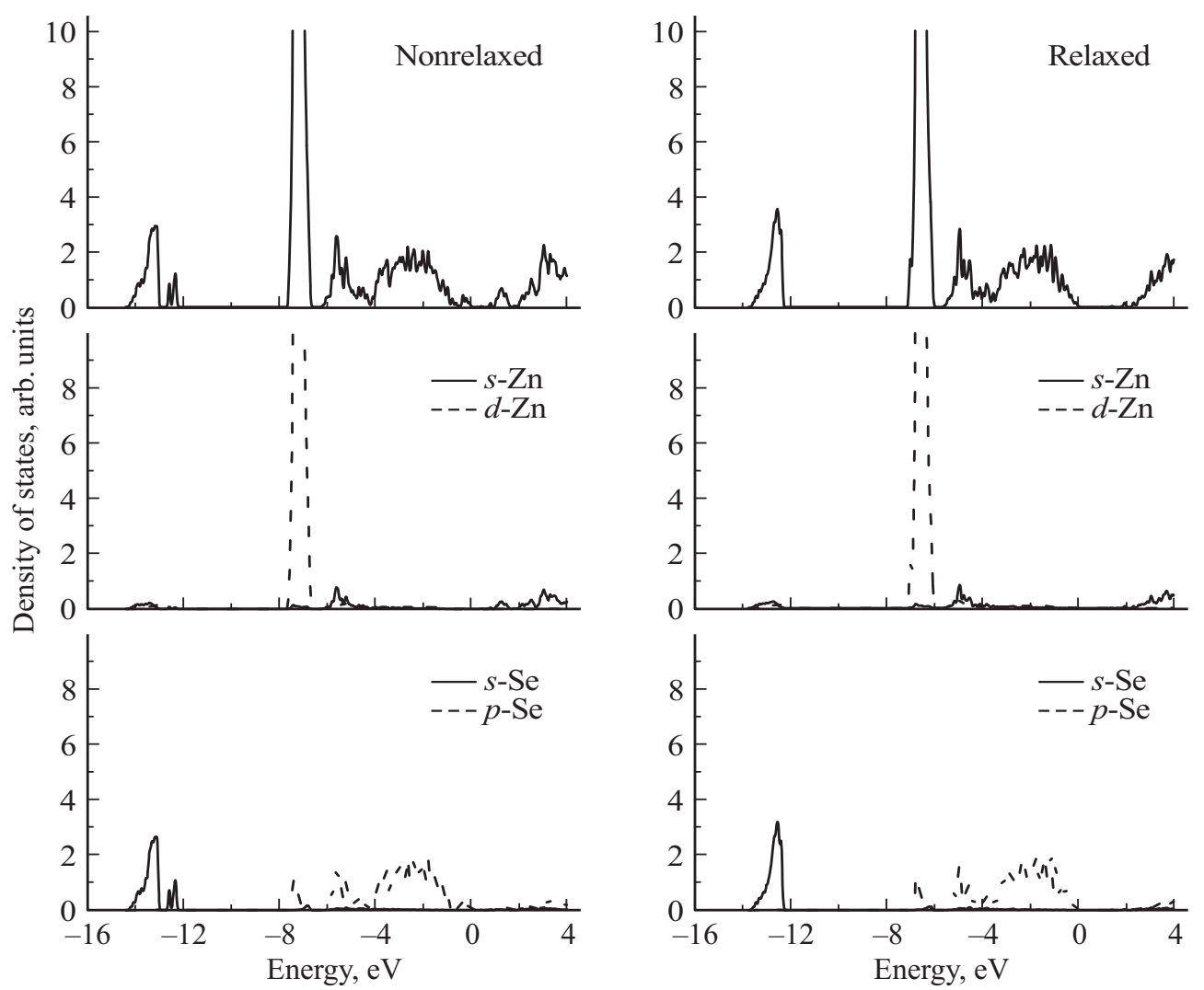

Рис. 16. Полная и $s$-, p-, $d$-DOS реконструированной поверхности (111) $\mathrm{ZnSe}$ без учета и с учетом релаксации. 


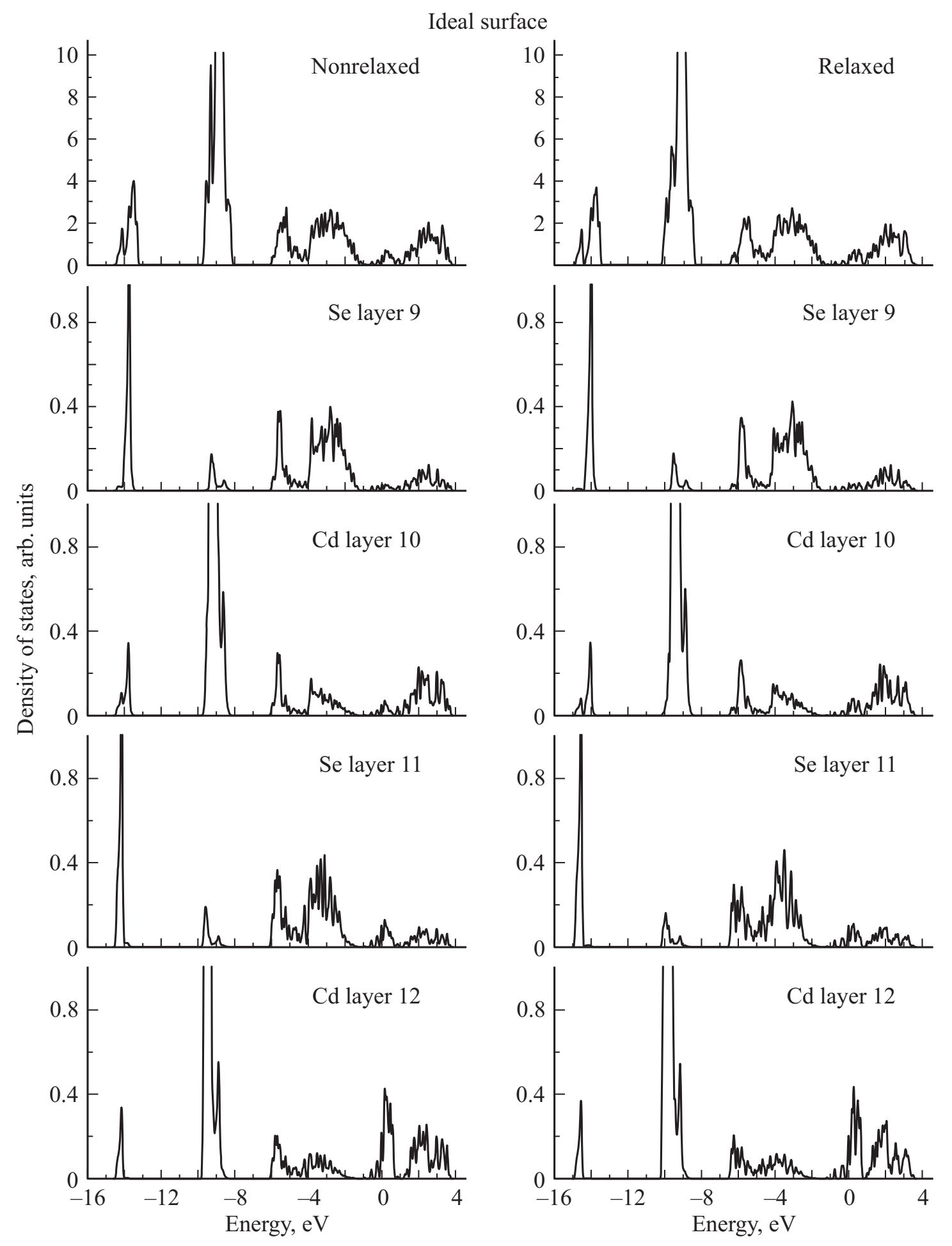

Рис. 17. Полная и послойная DOS идеальной поверхности (111) CdSe без учета и с учетом релаксации.

В то же время поверхностный слой $\mathrm{Cd} 12$ опустился на $0.8 \AA$ и занял место на уровне слоя Se11. В случае просто реконструкции максимальная сила, действующая на атом в поверхностном слое $\mathrm{Cd} 12$, равна $1.49 \mathrm{eV} / \AA$, а максимальная сила после релаксации действует в слое Se9 и уменьшается до $0.009 \mathrm{eV} / \AA$ - почти в 160 раз. Из рис. 7, $d$ видно, что в поверхностном слое до релаксации на все три атома действуют максимальные силы, равные $1.49 \mathrm{eV} / \AA$.

После проведения релаксации реконструированного слэба силы резко уменьшились практически до нуля.

Таким образом, в результате реконструкции/релаксации атомная структура пленки CdSe существенно изменилась. Эти изменения хорошо видны на рис. 8, 


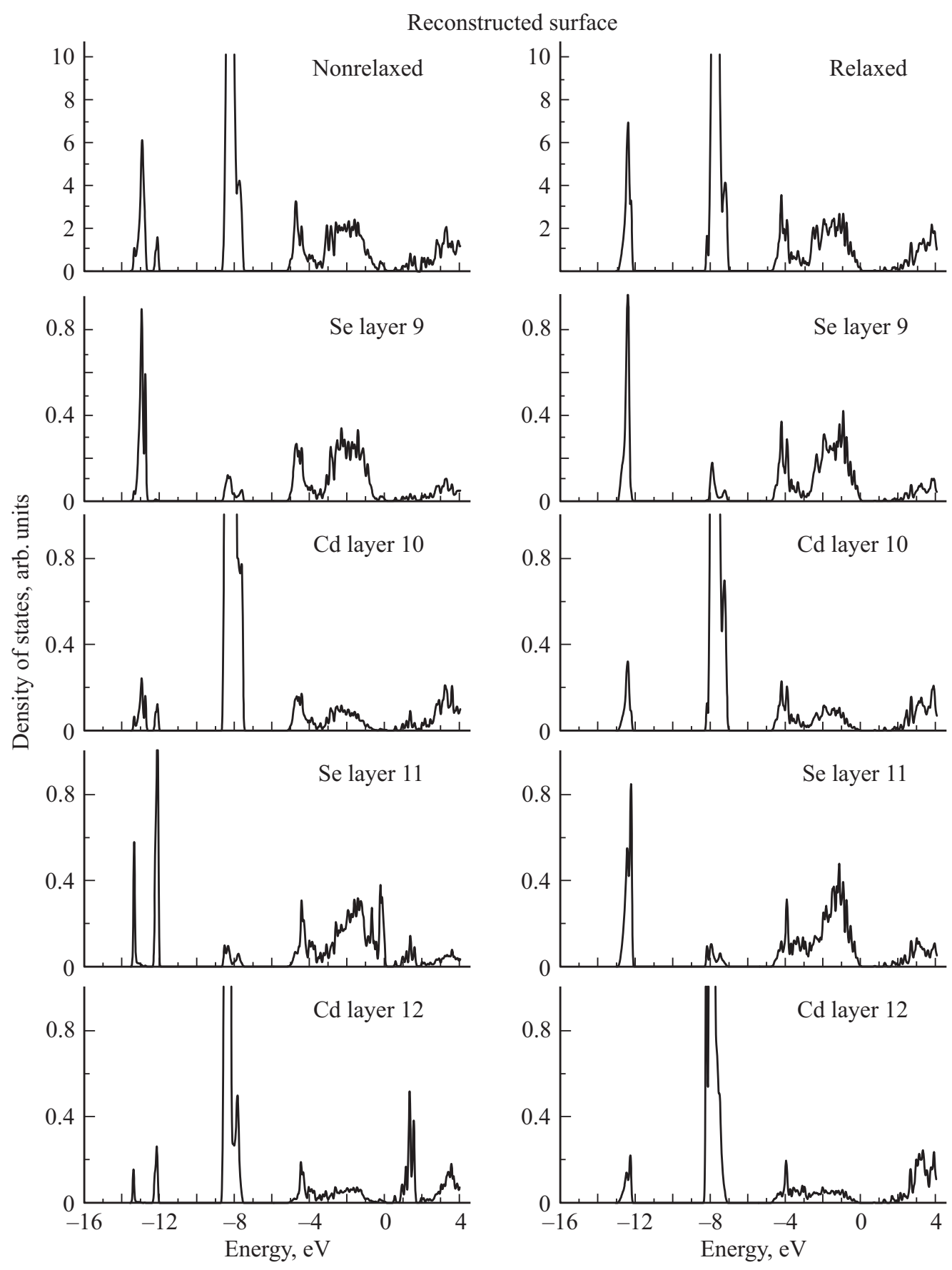

Рис. 18. Полная и послойная DOS реконструированной поверхности (111) CdSe без учета и с учетом релаксации.

где представлен вид пяти верхних атомных слоев реконструированной и реконструированной/релаксированной поверхностей.

\section{4. Зонная структура}

Зонная структура двенадцатислойных пленок $\mathrm{ZnSe}$ и $\mathrm{CdSe}$ с вакуумными промежутками вычислялась вдоль контура $\Gamma(0,0)-M(1 / 2,0)-K(1 / 3,1 / 3)-\Gamma(0,0)$ в двумерной зоне Бриллюэна. Изменение величины вакуум- ного промежутка в пределах нескольких элементарных ячеек не привело к заметным изменениям в зонной структуре и плотности электронных состояний.

4.1. $\mathrm{ZnSe}$. На рис. 9 и 10 представлены четыре варианта зонной структуры идеальной, релаксированной и реконструированной/релаксированной поверхностей $\mathrm{ZnSe}(111) \mathrm{A}-(2 \times 2)$.

Как было показано в разделе 1 , в исследуемой идеальной поверхности находится 217 валентных зон с учетом двух фиктивных атомов водорода. Происхождение четырех компактных групп уровней в валентной зоне авторы 


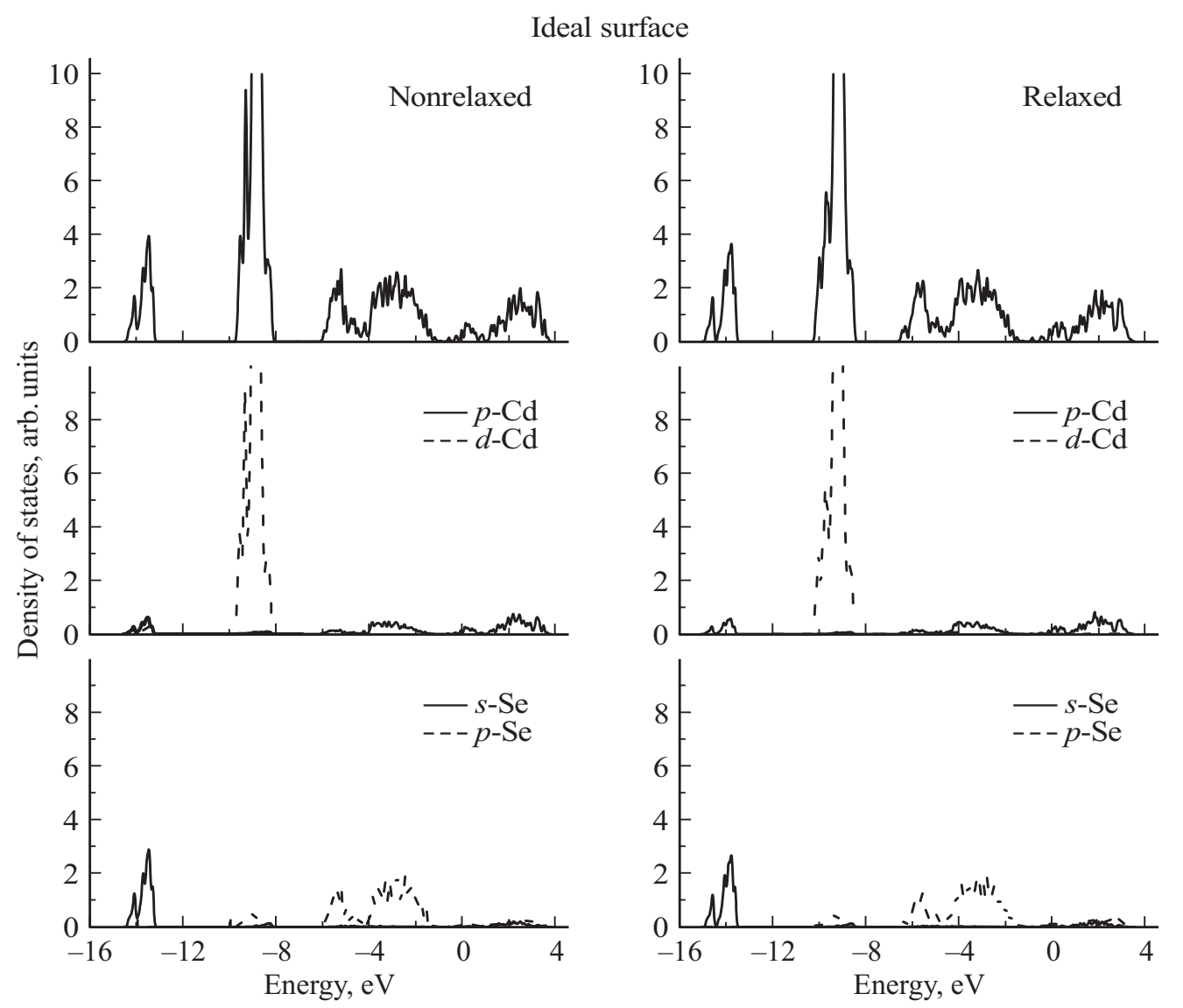

Рис. 19. Полная и $s-, p-, d$-DOS идеальной поверхности (111) CdSe без учета и с учетом релаксации.

подробно проанализировали в [21] на примере реконструированной поверхности CdTe, оканчивающейся кадмием. На рис. 9 в идеальной и релаксированной поверхностях 217-я валентная зона пересекает уровень Ферми, затем частично проходит через полосу проводимости и снова возвращается в валентную полосу. Таким образом, эти поверхности обладают металлическими свойствами.

В реконструированной поверхности с вакансией цинка в поверхностном слое (рис. 10) имеется 211 валентных зон. На рис. 10 вершина верхней валентной зоны совпадает с уровнем Ферми в точке $\Gamma$, ширина запрещенной зоны $E_{g}$ без учета релаксации равна $0.436 \mathrm{eV}$. Учет релаксации увеличивает $E_{g}$ до $1.43 \mathrm{eV}$. Таким образом, реальная поверхность $\mathrm{ZnSe}(111) \mathrm{A}(2 \times 2)$ является типичным прямозонным полупроводником.

4.2. CdSe. На рис. 11, 12 представлены четыре варианта зонной структуры идеальной, релаксированной и реконструированной/релаксированной поверхностей $\mathrm{CdSe}(111) \mathrm{A}-(2 \times 2)$.

Для пленки CdSe в случае идеальной и релаксированной поверхностей (рис. 11) зонные структуры похожи на результаты для пленки $\mathrm{ZnSe}$ (рис. 9). В случае реконструированной поверхности $\mathrm{CdSe}$, содержащей 211 валентных зон (см. рис. 12), вершина верхней валентной зоны и дно зоны проводимости смыкаются в точке Г, превращая слэб в полуметалл. Наконец, в случае реальной реконструированной поверхности с последующей релаксацией получился типичный полупроводник с $E_{g}=0.714 \mathrm{eV}$.

\section{5. Плотность состояний}

5.1. ZnSe. На рис. 13, 14 представлены результаты расчетов плотности электронных состояний (DOS) идеального, релаксированного, реконструированного и релаксированного после реконструкции слэбов $\mathrm{ZnSe}(111) \mathrm{A}-(2 \times 2)$.

Расчеты проводились на сетке точек $\mathbf{k} 6 \times 6 \times 1$. Энергетический интервал, в котором построены кривые, включал всю валентную зону, фундаментальную щель и часть зоны проводимости. Как и следовало ожидать, ширины запрещенных зон, определяемые из приведенных графиков плотности состояний и из графиков зонной структуры, совпадают.

Для каждого варианта слэба приведено по пять графиков. На верхнем графике приведена кривая полной DOS слэба, на остальных четырех графиках приведены DOS четырех верхних слоев Se9, Zn10, Se11 и Zn12, которые подверглись релаксации. Такое расположение позволяет наглядно проследить постепенное изменение DOS по мере приближения к верхнему поверхностному слою. Мы не будем подробно останавливаться на описании 


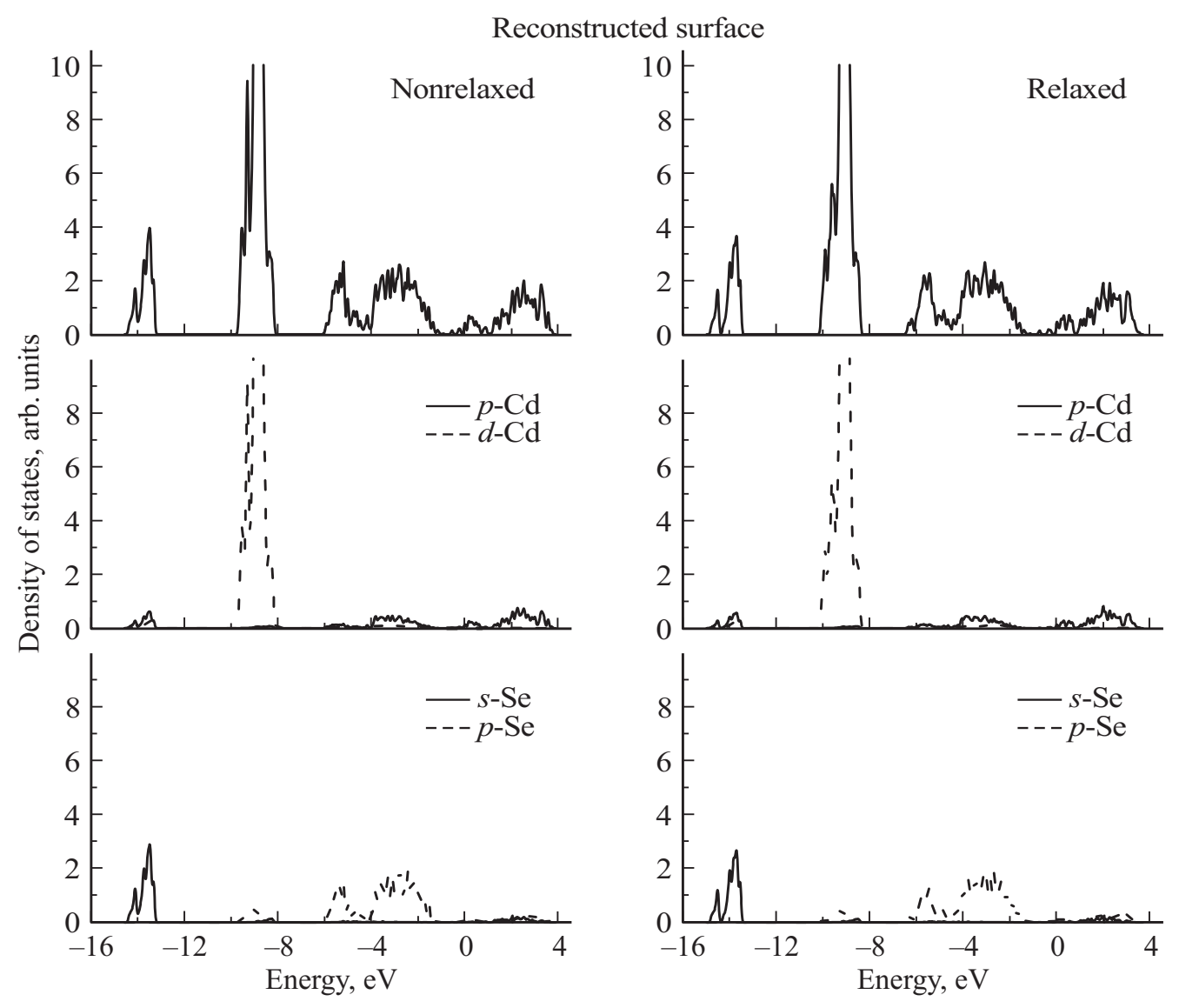

Рис. 20. Полная и $s-, p$-, $d$-DOS реконструированной поверхности (111) CdSe без учета и с учетом релаксации.

происхождения пиков DOS вдоль всей валентной полосы. Ясность вносят рис. 15, 16, на которых приведены вклады $s-, p$-, $d$-состояний валентных электронов в DOS идеального, релаксированного, реконструированного слэбов и реальной поверхности, т. е. релаксированной после реконструкции.

Отметим следующее. Кривые DOS идеального и релаксированного слэбов очень похожи по величине и расположению пиков. В поверхностном слое Zn12 в окрестности уровня Ферми выделяются пики оборванной связи, равные 0.371 и 0.318 arb.units в идеальном и релаксированном слэбах соответственно. В реконструированном слэбе уровень оборванной связи в поверхностном слое Zn12 сдвигается в запрещенную зону и уменьшается до 0.230 arb.units. Наконец, в реальной поверхности уровень оборванной связи теряется в зоне проводимости, и поверхность представляет собой типичный прямозонный полупроводник с $E_{g}=1.43 \mathrm{eV}$.

5.2. CdSe. На рис. 17, 18 представлены результаты расчетов DOS четырех вариантов слэбов $\mathrm{CdSe}(111) \mathrm{A}-(2 \times 2)$. На рис. 19, 20 приведены вклады $s$-, $p$-, $d$-состояний валентных электронов в DOS исследуемых слэбов.

Сравнительно небольшие пики, соответствующие $s H$-валентным электронам, встречаются по всей ширине валентной зоны и не приведены на графиках. Сравнение кривых плотности состояний на рис. 17-20 наглядно показывает, как постепенное преобразование поверхности от идеальной с объемными положениями атомов до реальной с релаксированными и расщепленными слоями, с учетверенной элементарной ячейкой и вакансией атома в верхнем поверхностном слое меняет кривые плотности состояний валентных электронов. В результате реальная поверхность (111) в CdSe является прямозонным полупроводником с $E_{g}=0.714 \mathrm{eV}$.

\section{6. Заключение}

С использованием данных $[11,12]$, впервые проведено теоретическое исследование и $a b$ initio расчеты электронной структуры четырех вариантов полярных поверхностей (111) в кристаллах $\mathrm{ZnSe}$ и $\mathrm{CdSe}$, заканчивающихся катионом: идеальной, релаксированной, реконструированной и релаксированной после реконструкции. В приближении слоистой сверхрешетки поверхность моделировалась системой тонких пленок (слэбов) толщиной двенадцать атомных слоев, периодически повторяющихся в направлении, перпендикулярном поверхности, и разделенных вакуумными промежутками $\sim 16 \AA$.

Для замыкания оборванных связей Se на противоположной стороне пленки добавляются четыре фиктивных атома водорода с зарядом 0.5 электрона каждый. 
$A b$ initio расчеты проводились с использованием программы QUANTUM ESPRESSO, основанной на DFT-LDA. В каждом из вариантов определены оптимальные силы, действующие на атомы, и равновесные координаты атомов $\mathrm{Zn}(\mathrm{Cd})$ и $\mathrm{Se}$ верхних „отпущенных“ четырех слоев. Положения атомов считаются полностью релаксированными, если силы, действующие на атомы, не превышают $0.02 \mathrm{eV} / \AA$.

Показано, что реконструкция расщепляет каждый из четырех верхних „отпущенных“ слоев на два слоя, содержащих два и один атом. Наши предыдущие результаты и экспериментальные данные показали, что такое расщепление присуще поверхности (111), оканчивающейся катионом, для большинства полупроводников $\mathrm{A}^{2} \mathrm{~B}^{6}$.

Рассчитаны зонные структуры четырех вариантов слэба и проанализировано влияние релаксации и реконструкции на особенности поведения зоны проводимости и валентной зоны, а также положение уровня Ферми. В случае реальной (реконструкция/релаксация) поверхности получился типичный прямозонный полупроводник. Рассчитаны полные и послойные плотности состояний валентных электронов для четырех верхних слоев: $9,10,11,12$. Приведенные рисунки наглядно демонстрируют постепенное изменение величины и расположения поверхностных структур по шкале энергий при переходе от слоя к слою и от одного вида поверхности к другому.

Полученные значения ширины запрещенной зоны следует рассматривать как оценочные, так как известно, что расчетные методы, базирующиеся на DFT, занижают ширину запрещенной зоны в полупроводниках и диэлектриках (см., например [26,27]). Для решения проблемы получения корректной величины ширины запрещенной зоны с конца 90-х годов начали использовать гибридные обменно-корреляционные функционалы, которые включают нелокальный обменный функционал Хартри-Фока, а также обменно-корреляционные функционалы DFT. Использование гибридных функционалов приводит к сдвигу валентных состояний в сторону низких энергий, тогда как незанятые состояния сдвигаются в сторону высоких энергий, увеличивает запрещенную щель, при этом структура зон остается практически без изменений [28-31]. Поскольку в настоящей работе проведено исследование последовательного изменения свойств зонной структуры поверхности, которые имеют место в ряду: идеальная нерелаксированная, идеальная релаксированная, реконструированная нерелаксированная и релаксированная после реконструкции (т.е. реальная) поверхности, то, в свете сказанного выше, наши результаты являются достоверными.

\section{Список литературы}

[1] J.P. LaFemina. Surf. Sci. Rep. 16, 133 (1992).

[2] G.P. Srivastava. Theoretical modeling of semiconductor surfaces. World Scientific, Singapore, New Jersey, London, Hong Kong (1999). P. 201-205.

[3] J.A. Venables. Introduction to Surface and Thin Film Processes. Cambridge university press, Arizona State University and University of Sussex (2003). 392 p.
[4] A. Groß. Theoretical Surface Science. Springer, Berlin, Heidelberg (2009). $342 \mathrm{p}$.

[5] K. Hermann. Crystallography and Surface Structure. An Introduction for Surface Scientists and Nanoscientists. WileyVCH Verlag \& Co, Weinheim (2011). 294 p.

[6] II-VI Semiconductor Compounds / Ed. M. Jain. World Scientific, Singapore (1993). 604 p.

[7] R.W. Birkmire, E. Eser. Ann. Rev. Mater. Sci. 27, 625 (1997).

[8] M.A. Haase, J. Qiu, J.M. DePuydt, H. Cheng. Appl. Phys. Lett. 59, 1272 (1991).

[9] N. Nakayama, S. Itoh, T. Ohata, K. Nakano, H. Okuyama, M. Ozawa, A. Ishibashi, M. Ikeda, Y. Mori. Electron. Lett. 29, 2194 (1993).

[10] A. Salokatve, H. Jeon, J. Ding, M. Hovinen, A.V. Nurmikko, D.C. Grillo, Li He, J. Han, Y. Fan, M. Ringle, R.L. Gunshor, G.C. Hua, N. Otsuka. Electron. Lett. 29, 2192 (1993).

[11] D.J. Chadi. J. Vac. Sci. Technol. A 4, 944 (1986).

[12] L. Zhu, K.L. Yao, Z.L. Liu, Y.B. Li. J. Phys.: Condens. Matter 21, 095001 (2009).

[13] L. Plucinski. Bulk and Surface Electronic Structure of Gallium Nitride and Zinc Selenide. Dissertation, Hamburg (2002). $126 \mathrm{p}$.

[14] W. Weigand, A. Müller, L. Kilian, O. Bunk, T. Schallenberg, P. Bach, L. Molenkamp, W. Faschinger, R.L. Johnson, C. Kumpf, E. Umbach. Hasylab Annual Report 2002. Pt 1. HASYLAB, Hamburg (2003).

[15] D. Olguin, R. Baquero. Rev. Mexic. Fis. 49, 1 (2003).

[16] L. Plucinski, R.L. Johnson, A. Fleszar, W. Hanke, W. Weigand, C. Kumpf, C. Heske, E. Umbach, T. Schallenberg, L.W. Molenkamp. Phys. Rev. B 70, 125308 (2004).

[17] L. Plucinski, W. Weigand, C. Kumpf, C. Heske, R. Kosuch, T. Schallenberg, L.W. Molenkamp, E. Umbach, R.L. Johnson. Surf. Sci. 585, 95 (2005).

[18] Y. Yu, J. Zhou, H. Han, C. Zhang, T. Cai, C. Song, T. Gao. J. All. Comp. 471, 492 (2009).

[19] A. Rubio-Ponce, D. Olguin. J. Physics: Conf. Ser. 574, 012118 (2015).

[20] J. Zhou, B.G. Sumpter, P.R C. Kent, J. Huang. Appl. Mater. Interfac. 7, 1458 (2015).

[21] В.Л. Бекенев, С.М. Зубкова. ФТТ 57, 1830 (2015).

[22] В.Л. Бекенев, С.М. Зубкова. ФТП 51, 26 (2017).

[23] P. Giannozzi, S. Baroni, N. Bonini, M. Calandra, R. Car, C. Cavazzoni, D. Ceresoli, G.L. Chiarotti, M. Cococcioni, I. Dabo, A.D. Corso, S. Fabris, G. Fratesi, S. de Gironcoli, R. Gebauer, U. Gerstmann, C. Gougoussis, A. Kokalj, M. Lazzeri, L. Martin-Samos, N. Marzari, F. Mauri, R. Mazzarello, S. Paolini, A. Pasquarello, L. Paulatto, C. Sbraccia, S. Scandolo, G. Sclauzero, A.P. Seitsonen, A. Smogunov, P. Umari, R.M. Wentzcovitch. J. Phys.: Condens. Matter. 21, 395502 (2009).

[24] K. Shiraishi. J. Phys. Soc. Jpn. 59, 3455 (1990).

[25] Landolt-Bornstein / Ed. O. Madelung. New Series III. 22. Springer, Berlin (1987).

[26] J.P. Perdew. Int. J. Quantum Chem. 28, S19, 497 (1985).

[27] J.L. Martins. In: Density Functional Theory / Ed. P. Geerlings, F. de Proft, W. Langenaeker. VUB University Press, Brussels (1999). P. 217-226.

[28] S. de Lazaro, E. Longo, J.R. Sambrano, A. Beltran. Surf. Sci. 552, 149 (2004).

[29] S. Piskunov, E. Heifets, R.I. Eglitis, G. Borstel. Comp. Mater. Sci. 29, 165 (2004).

[30] J. Muscat, F. Wander, N.M. Harrison. Chem. Phys. Lett. 342, 397 (2001).

[31] E. Heifets, R.I. Eglitis, E.A. Kotomin, J. Maier, G. Borstel. Surf. Sci. 513, 211 (2002). 